\title{
Accurate hybrid template-based and MR- based attenuation correction using UTE images for simultaneous PET/MR brain imaging applications
}

Jakub Baran ${ }^{1,2,3^{*}}$ (D), Zhaolin Chen ${ }^{1,4}$, Francesco Sforazzini ${ }^{1}$, Nicholas Ferris ${ }^{1,5}$, Sharna Jamadar ${ }^{1,6,7}$, Ben Schmitt ${ }^{8}$, David Faul ${ }^{9}$, Nadim Jon Shah ${ }^{1,10}$, Marian Cholewa ${ }^{2}$ and Gary F. Egan ${ }^{1,6,7}$

\begin{abstract}
Background: Attenuation correction is one of the most crucial correction factors for accurate PET data quantitation in hybrid PET/MR scanners, and computing accurate attenuation coefficient maps from MR brain acquisitions is challenging. Here, we develop a method for accurate bone and air segmentation using MR ultrashort echo time (UTE) images.

Methods: MR UTE images from simultaneous MR and PET imaging of five healthy volunteers was used to generate a whole head, bone and air template image for inclusion into an improved MR derived attenuation correction map, and applied to PET image data for quantitative analysis. Bone, air and soft tissue were segmented based on Gaussian Mixture Models with probabilistic tissue maps as a priori information. We present results for two approaches for bone attenuation coefficient assignments: one using a constant attenuation correction value; and another using an estimated continuous attenuation value based on a calibration fit. Quantitative comparisons were performed to evaluate the accuracy of the reconstructed PET images, with respect to a reference image reconstructed with manually segmented attenuation maps.

Results: The DICE coefficient analysis for the air and bone regions in the images demonstrated improvements compared to the UTE approach, and other state-of-the-art techniques. The most accurate whole brain and regional brain analyses were obtained using constant bone attenuation coefficient values.

Conclusions: A novel attenuation correction method for PET data reconstruction is proposed. Analyses show improvements in the quantitative accuracy of the reconstructed PET images compared to other state-of-the-art AC methods for simultaneous PET/MR scanners. Further evaluation is needed with radiopharmaceuticals other than FDG, and in larger cohorts of participants.
\end{abstract}

Keywords: PET/MR, Attenuation correction, Brain, UTE

\footnotetext{
* Correspondence: jakubbaran92@gmail.com

${ }^{1}$ Monash Biomedical Imaging, Monash University, Melbourne, Australia

${ }^{2}$ Department of Biophysics, Faculty of Mathematics and Natural Sciences,

University of Rzeszow, Rzeszow, Poland

Full list of author information is available at the end of the article
}

(c) The Author(s). 2018 Open Access This article is distributed under the terms of the Creative Commons Attribution 4.0 International License (http://creativecommons.org/licenses/by/4.0/), which permits unrestricted use, distribution, and reproduction in any medium, provided you give appropriate credit to the original author(s) and the source, provide a link to the Creative Commons license, and indicate if changes were made. The Creative Commons Public Domain Dedication waiver (http://creativecommons.org/publicdomain/zero/1.0/) applies to the data made available in this article, unless otherwise stated. 


\section{Background}

Positron Emission Tomography and Magnetic Resonance (PET/MR) scanners allow for simultaneous data acquisition of both modalities and are a very powerful tool for diagnostic and research imaging. Metabolic information obtained from PET scans, combined with the excellent anatomical and functional contrast derived from MRI examinations, provides new possibilities in medical imaging research. In the oncological applications [1-3], where excellent MRI soft tissue contrast distinguishes between soft tissues better than Computed Tomography (CT). PET/ MR has also shown promising results in application to neurological studies [4-8]. An additional advantage of $\mathrm{PET} / \mathrm{MR}$ imaging, compared to PET/CT, is the absence of radiation exposure from $\mathrm{CT}$ examination. However, there are a number of important limitations for simultaneous $\mathrm{PET} / \mathrm{MR}$ imaging, which need further work including accurate attenuation correction of the PET data [9]. This factor is crucial for quantitative PET data analysis in PET/ MR scanners, and still remains an active area of methodological development.

Due to the MRI signal properties, the extraction of attenuation coefficients for $511 \mathrm{keV}$ photons detected using PET is not as straightforward as it is in the case of CT [10-12]. The MRI signal depends on many factors, particularly on the proton distribution and relaxation time of the tissue, whereas the CT signal is electron-density and X-ray spectrum dependant. For the most commonly used sequences in MRI, the signal from cortical bone has a similar intensity as that from air, due to the very short T2* relaxation time. Accurate attenuation factor maps are crucial for precise PET data reconstruction, particularly for neurological imaging applications. Significant image artifacts and spatial biases in PET images are found in brain tissue adjacent to the cortical bone, which absorb positron annihilation photons much more strongly than soft tissues. Difficulties in separating cortical bone from air (and/or ignoring bone) when the attenuation correction is based on MR data also contribute to the artifacts $[13,14]$. All of these factors necessitate the development of an improved technique for MR-based attenuation correction, especially for neurological applications where demands on the accuracy of tracer quantitation are high.

Currently the two commercially available simultaneous PET/MR scanners have a number of attenuation correction techniques. The Biograph mMR (Siemens Healthcare GmbH, Erlangen, Germany) provides three different techniques, including a method that employs two-point Dixon water and fat imaging and image segmentation [15]. This sequence is not recommended for brain applications, due to an underestimation of PET uptake results [16]. A second technique segments images into bone, soft tissue and air using a dual ultrashort echo time sequence (dUTE) to capture the signal from bone, whilst a third atlas-based technique produces attenuation correction maps using only Dixon images $[17,18]$. The Signa PET/MRI (GE Healthcare, Waukesha, WI, USA) offers a 4-compartment class whole-body atlas-based technique [19].

There are three main groups of attenuation correction methods: PET based; MR segmentation based; and MR-CT atlas/template based. The main concept behind PET based methods is to optimise the reconstruction based methods to reconstruct attenuation and activity simultaneously (MLAA) [20]. Extensions of this method have been developed for both time-of-flight (TOF) $[21,22]$ and non-TOF [23] applications. Due to the high computational demands MLAA based techniques are not practical for clinical applications. One of the most interesting approaches in recent years utilises the background radiation of PET scintillators for the simultaneous acquisition of transmission and emission data [24].

Segmentation-based methods generate attenuation correction maps by relying only on MR images. The attenuation maps are typically segmented into several tissue types and specific attenuation coefficient values are assigned to them. The most commonly used images for tissue segmentation are UTE images [25-29]. In order to assign the continuous attenuation coefficients values for bone, R2* map approaches are typically used, calculated as follows [26, 29]: $R 2^{*}=\frac{\log I_{1}-\log I_{2}}{T E_{2}-T E_{1}}$, where $I_{1}$ and $I_{2}$ are the first and second echo images and $T E_{1}, T E_{2}$ are the echo times. Moreover, due to rapidly growing computational capabilities, increasingly sophisticated methods such as machine learning and deep learning are being employed for tissue segmentation [30-33]. A number of methods also use T1 images [34] or improved/modified UTE sequences [35-38].

The MR-CT atlas/template-based methods produce pseudo-CT images based on CT-MR databases. Subject images are typically co-registered to the atlas or template, which allows for the assignment of continuous attenuation coefficient values for all voxels, but does not take into account subject-specific variability. Typically, T1w/T2w images are used for this purpose [39-44], with UTE images used rarely $[45,46]$. There are also methods that combine atlas based approaches with machine learning or probabilistic methods [47-49]. A more detailed description of the existing attenuation correction methods can be found in $[50,51]$.

The aforementioned attenuation correction techniques in the literature are usually compared with the vendor's techniques and a reference CT-based attenuation map. Detailed comparisons between attenuation correction techniques developed by academic groups and by one vendor have been undertaken by $[52,53]$. Cabello and co-workers performed an assessment of four well established methods and the Siemens's UTE method in comparison to a 
reference CT-based attenuation correction map using data from a cohort of 15 patients who have been administered ${ }^{18}$ F-FDG. A more comprehensive comparison was undertaken in the [53] paper, including evaluation of eleven independent techniques. Three different cohorts from two centres with the overall number of 359 patients were retrospectively analysed. The best overall performance was achieved with atlas-based and segmentation-based techniques. Despite the large number of techniques that were included in the study and the number of patients that were examined, the authors conclusions require further investigation due to the use of CT images as a reference and the necessity to investigate the techniques in younger patients and in patients with focal lesions.

In this work, we introduce a new segmentation technique segmented UTE (sUTE) to generate improved attenuation correction maps for neurological applications. A UTE template based on manual segmentation of the head image is used to segment air, bone and tissue. Two different methods are used to generate attenuation correction maps. The first method assigns fixed attenuation coefficients within a tissue type, and the second method employs subject-specific information from the R2: map to estimate continuous attenuation coefficients for bone. Whilst these methods appear to be similar to other published techniques [26, 41], there are substantial differences between the published techniques and the sUTE methods. The segmentation step with sUTE is fully based on the first echo UTE images, whereas the RESOLUTE technique [26] relies on dual echo UTE images and does not employ Gaussian Mixture Models. The technique developed by Anazodo and co-workers [41] utilises Dixonbased attenuation maps for soft tissue and air, and segments the T1 weighted image to extract a bone mask. The segmentation also involves the Gaussian Mixture Models, albeit tissue probabilistic maps covering only the standard MNI (Montreal Neurological Institute) template space, and does not cover the whole head as in the sUTE method. In this study, we constructed attenuation correction maps based on manually segmented MR images as a reference and investigated the differences between CT-based attenuation correction maps and our manual segmentation based attenuation maps. We also studied the impact of different attenuation coefficient assignments for white matter (WM), grey matter (GM) and cerebrospinal fluid (CSF) on the intensity values in reconstructed PET images. The results from the proposed methods are compared to the vendor UTE-based method and other state-of-the-art methods.

\section{Methods}

PET-MRI datasets were acquired at Monash Biomedical Imaging, Monash University, Melbourne, Australia, between July 2016 and November 2016. All scans were performed using a fully-integrated PET/MR scanner (Siemens Biograph mMR, Siemens Healthcare, Erlangen, Germany with the software version VB20P). All examinations were approved by the Monash University Human Research Ethics Committee.

CT-MRI datasets were acquired at Institute of Neuroscience and Medicine, Forschungszentrum Juelich $\mathrm{GmbH}$, Juelich, Germany and PET/CT Radiologie/Nuklearmedizine UKD Dusseldorf. All MRI scans were performed with a $3 \mathrm{~T}$ MRI scanner (MAGNETOM Trio Tim, Siemens Health care, Erlangen, Germany with the software version VB13A) equipped with Siemens BrainPET inserts. All CT scans were acquired with two scanners: Philips GEMINI GXL16 PET/CT and Siemens Biograph 128 PET/CT.

\section{Healthy subjects}

The group of subjects examined using PET consisted of five healthy participants with $\left[{ }^{18} \mathrm{~F}\right]-\mathrm{FDG}$ intravenously administrated at a constant infusion rate of $36 \mathrm{ml} /$ hour for $95 \mathrm{~min}$. Additionally, one healthy female participant was scanned with the same MRI protocol, but without administration of the PET radiopharmaceutical. The MRI images of this participant were used to create the UTE1 (UTE first echo image) template used for bone and air segmentation. The group of five participants who undertook the PET examination are referred to as the PET group throughout this paper.

The group of subjects examined using CT and MRI consisted of four (76.8 \pm 6.7 years) healthy participants who undertook PET/CT and PET/MR acquisition. This group is referred to as the $\mathrm{CT}$ group throughout this paper.

\section{Imaging protocol \\ PET imaging protocol in the PET group}

Information about the participants in the PET group is given in Table 1. 95-min PET data acquisition started at the same time as radiotracer administration. The PET data were acquired in the list mode, and the one frame 95-min data reconstruction was performed offline using e7tools software provided by Siemens. Ordinary Poisson Ordered Subset Expectation Maximization (OP-OSEM) algorithm with Point Spread Function (PSF) correction was used with 3 iterations, 21 subsets and $344 \times 344 \times 127$ (voxels size: $2.09 \times 2.09 \times 2.03 \mathrm{~mm}^{3}$ ) reconstruction matrix size. A 5-mm Gaussian post-filtering was applied to the

Table 1 PET group information ${ }^{a}$

\begin{tabular}{llll}
\hline Radiotracer & $\begin{array}{l}\text { Age average } \\
\text { (SD) in years }\end{array}$ & Female/male & $\begin{array}{l}\text { Injected radiotracer } \\
\text { (SD) activity in MBq }\end{array}$ \\
\hline$\left[{ }^{18} \mathrm{~F}\right]$-FDG & $33(9.9)$ & $4 / 1$ & $82(29)$ \\
\hline
\end{tabular}

${ }^{a}$ Table does not include one additional participant who underwent MRI scan without radiotracer administration 
final reconstructed images. The PET protocol was the same for all studies.

\section{MRI imaging protocol}

Three sequences were acquired simultaneously with the PET acquisition for the PET group to use for evaluating the attenuation correction methods. The dUTE-AC sequence was acquired with the following parameters: echo time 1 (TE1)/echo time 2 (TE2)/repetition time $(\mathrm{TR})=0.07 / 2.46 / 11.94 \mathrm{~ms}$, flip angle $10^{\circ}$, voxel size: $1.56 \times 1.56 \times 1.56 \mathrm{~mm}^{3}$, matrix size: $192 \times 192 \times 192$. The dual-echo Dixon sequence was acquired with TE1/TE2/ $\mathrm{TR}=1.23 / 2.46 / 3.6 \mathrm{~ms}$, flip angle: $10^{\circ}$, voxel size: $2.08 \times$ $2.08 \times 2.34 \mathrm{~mm}^{3}$, matrix size: $192 \times 126 \times 128$. Lastly, a T1w MPRAGE was acquired with $\mathrm{TE} / \mathrm{TR}=2.34$ / $1640 \mathrm{~ms}$, flip angle: $8^{\circ}$, voxel size: $1 \times 1 \times 1 \mathrm{~mm}^{3}$, matrix size: 256x256x176. The overall MR acquisition time was approximately $9 \mathrm{~min}$.

The dUTE sequence was acquired with the following parameters: TE1/TE2/TR $=0.07 / 2.46 / 200 \mathrm{~ms}$, flip angle $15^{\circ}$,voxel size: $1.67 \times 1.67 \times 1.67 \mathrm{~mm}^{3}$, matrix size: $192 \times 192 \times 192$ for the subjects included within the CT group.

\section{CT imaging protocol in the CT group}

The protocol parameters for each subject in CT group are presented in Table 2.

\section{UTE1 template and probabilistic maps creation}

The whole head template creation process required several steps. Ground truth air, soft tissue, and bone masks were obtained by manual segmentation of both echo UTE images under the supervision of a clinical radiologist (NF). Images of five participants (four from the PET group, and one with only MRI data) were used. The UTE template ( $\left.\mathrm{UTE}_{\text {template }}\right)$ was created using the first echo UTE images, by applying the combination of affine and non-linear registration transformations (ANTS, PICSL, Philadelphia, PA). The $\mathrm{UTE}_{\text {template }}$ space was in the subject space. All manually segmented masks were subsequently transformed to the template space by applying corresponding transformation matrices; trilinear interpolation was used. The tissue probability maps (TPM) were then averaged within tissue types to create temporary TPM. Due to the constraint that the sum of all tissue probabilities for a single voxel must be equal to unity, all voxels were normalized.
Final air $\left(\mathrm{TPM}_{\mathrm{air}}\right)$ and bone $\left(\mathrm{TPM}_{\mathrm{bone}}\right)$ tissue probability maps were obtained.

To generate GM, WM, CSF and soft tissue probabilistic maps, the SPM12 toolbox (Statistical Parametric Mapping, Wellcome Trust Centre for Neuroimaging) [54] was used. Segmentation of the UTE1 images was processed using probabilistic tissue templates provided in SPM12. Bias normalisation was performed prior to segmentation. Using the corresponding transformations, grey matter, white matter, cerebrospinal fluid and soft tissue probability, maps were warped into the template space, averaged and normalised with respect to the air and bone probability maps to obtain final GM, WM, CSF and soft tissue probability maps. If the sum of all TPM within the voxel was greater than 1 , the values for air and bone were not changed, but the remaining tissues were rescaled correspondingly. The tissue probability maps for CSF covered only MNI space, and did not include CSF in the spinal region below that space. The tissue probability maps are shown in Fig. 1.

\section{Attenuation correction map generation}

The first echo UTE volume was non-rigidly registered to

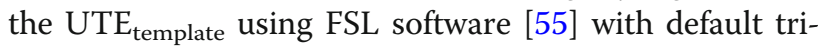
linear interpolation and Correlation Ratio cost function. The SPM12 software was then used to perform the segmentation (Gaussian Mixture Model) using $\mathrm{UTE}_{\text {template }}$ tissue probability maps as a priori information.

All voxels in the resulting air probability map above 0.1 were identified as air and constant attenuation correction factors equal to $0 \mathrm{~cm}^{-1}$ were assigned. Resulting bone probability maps had a threshold of 0.2 and all values above that threshold were identified as bones. Bone attenuation factors were calculated in two ways: (i) a third order polynomial transformation [26] was used to map R2* intensity vales to attenuation coefficients (AC $\left.C_{\text {sUTEcont }}\right)$; and (ii) a constant value of $0.151 \mathrm{~cm}^{-1}$ was assigned $\left(\mathrm{AC}_{\mathrm{sUTEfix}}\right)$. The attenuation maps were then smoothed with a 2-mm kernel Gaussian filter.

For the remaining soft tissue voxels, a $0.100 \mathrm{~cm}^{-1}$ value was assigned. If the template field-of-view (FOV) was smaller than the participant FOV (the template covers only the part of the neck), the vendor provided UTE-based attenuation map values that were used to fill the remaining FOV. The workflow of the attenuation maps generation is depicted in Fig. 2.

Table $2 \mathrm{CT}$ acquisition setups in the $\mathrm{CT}$ group

\begin{tabular}{llllll}
\hline Participant no. & Scanner & X-Ray Tube Voltage $[\mathrm{kVp}]$ & X-Ray Tube Current[mA] & Matrix size & Voxel size $\left[\mathrm{mm}^{3}\right]$ \\
\hline 01 & Phillips GEMINI GXL16 & 120 & 57 & $512 \times 512 \times 320$ & $0.488 \times 0.488 \times 0.750$ \\
02 & Siemens Biograph 128 & 120 & 55 & $512 \times 512 \times 229$ & $0.482 \times 0.482 \times 1.000$ \\
03 & Siemens Biograph 128 & 120 & 60 & $512 \times 512 \times 321$ & $0.482 \times 0.482 \times 0.600$ \\
04 & Siemens Biograph 128 & 120 & 60 & $512 \times 512 \times 380$ & $0.494 \times 0.494 \times 0.600$ \\
\hline
\end{tabular}




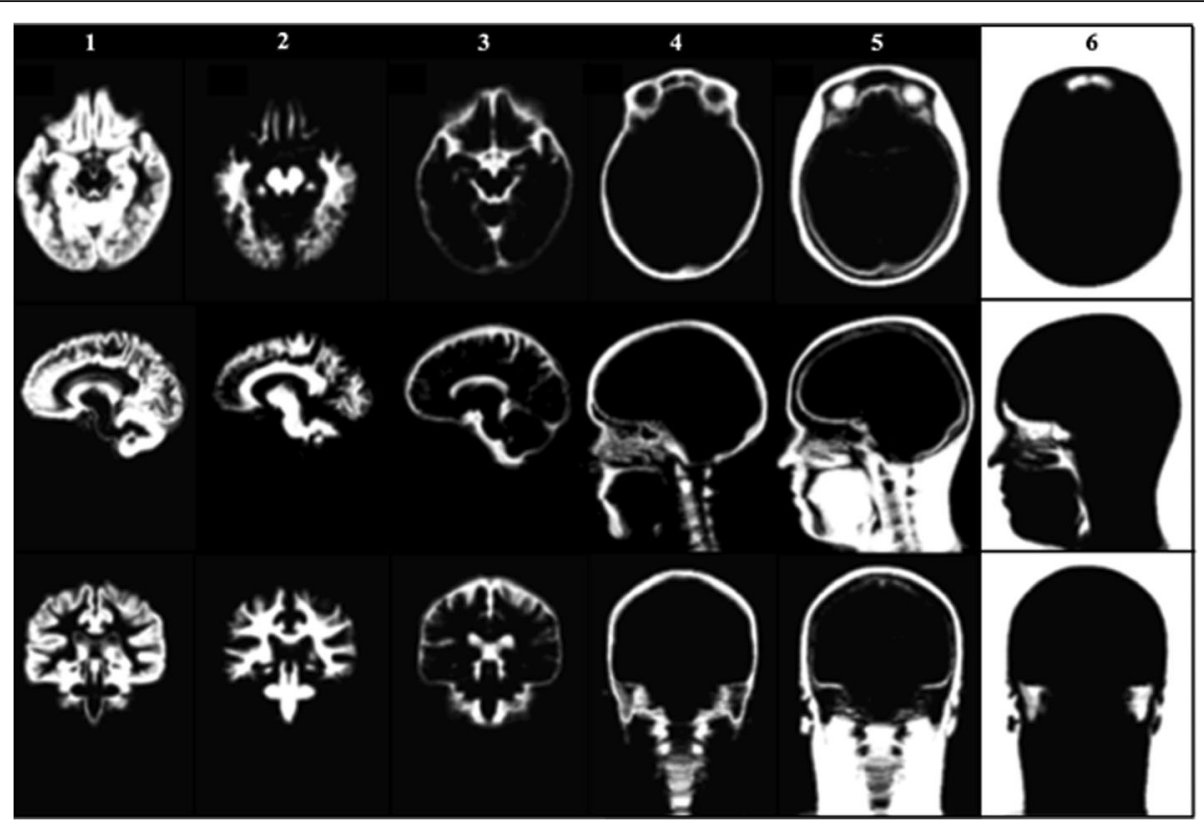

Fig. 1 Tissue probabilistic maps used in the study. Tissue probabilistic maps for grey matter (column 1), white matter (column 2), cerebrospinal fluid (column 3), bone (column 4), soft tissue (column 5) and air (column 6) in axial (top), sagittal (centre) and coronal (bottom) view

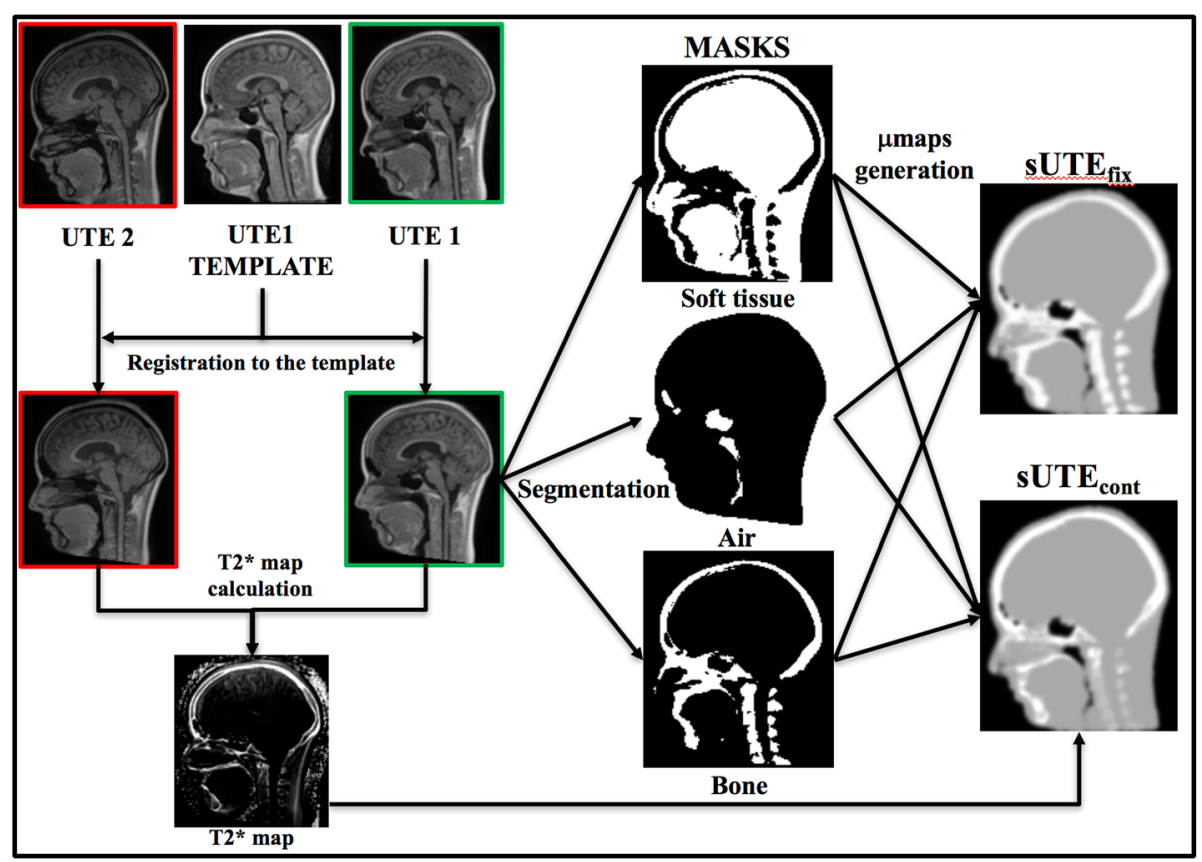

Fig. 2 Attenuation maps generation workflow. Both UTE images are rigidly registered to the UTE template. Segmentation into three tissue classes (soft tissue, air, bone) is performed using first echo UTE image registered to the UTE template. SUTE maps are then generated by assigning attenuation coefficients to all classes with respect to the masks, 3D 2-mm Gaussian smoothing and warping back to the subject space. Attenuation coefficients are fixed for soft tissue and air $\left(0.1 \mathrm{~cm}^{-1}\right.$ and $0 \mathrm{~cm}^{-1}$, respectively), whereas for bones can be either fixed - equal $0.151 \mathrm{~cm}^{-1}\left(\mathrm{sUTE}_{\mathrm{fix}}\right)$ or continuous - based on $\mathrm{R}^{*}$ map (sUTE $\left.{ }_{\text {cont }}\right)$ 
Four AC maps were computed and compared as follows:

1. The manually segmented reference attenuation maps had the following attenuation coefficient values assigned for bone, soft tissue, and air respectively: $0.151 \mathrm{~cm}^{-1}, 0.1 \mathrm{~cm}^{-1}$ and $0 \mathrm{~cm}^{-1}$ $(\mathrm{AC}$ ref $)$.

2. CT-based attenuation maps were computed by converting HU (Hounsfield Units) maps to the $511 \mathrm{keV}$ attenuation coefficient maps $\left(\mathrm{AC}_{\mathrm{CT}}\right)$ [11].

3. The pseudo-CT method proposed by Burgos et al. [39] $\left(\mathrm{AC}_{\mathrm{UCL}}\right)$ was based on non-rigid co-registration and similarity measurements between each participant and 41 subjects from a T1w-CT image database. The $\mathrm{CT}$ images were generated using the open source software described in [56], converted to attenuation coefficients and smoothed with a 2-mm Gaussian filter. The T1 weighted images were used as inputs. The mean attenuation coefficients were $0.161 \mathrm{~cm}^{-1}$ and $0.096 \mathrm{~cm}^{-1}$ for bone and soft tissue, respectively.

4. The UTE-based (vendor-provided) technique was also evaluated $\left(\mathrm{AC}_{\mathrm{UTE}}\right)$.

\section{Methods for comparing different attenuation correction maps}

\section{Attenuation correction maps comparison}

The segmentation accuracy of $\mathrm{AC}_{\mathrm{UTE}}, \mathrm{AC}_{\mathrm{UCL}}$ and the proposed method was evaluated using the DICE coefficient, false-positive (FP) and false-negative (FN) rates with respect to the manual segmentation. In case of $\mathrm{AC}_{\mathrm{UCL}}$, voxels lower than $-500 \mathrm{HU}$ were classified as air, and voxels higher than $300 \mathrm{HU}$ were classified as bone [29]. The classification for $\mathrm{AC}_{\mathrm{UTE}}$ was performed by setting all voxels with a value equal to $0.151 \mathrm{~cm}^{-1}$ as bone, whereas voxels with a value of $0 \mathrm{~cm}^{-1}$ were assigned as air. A quantitative analysis was conducted for the head region (covering brain and nasal cavities). The DICE coefficient was determined as follows:

$$
\text { Dice coefficient }=\frac{2 \cdot\left(A C_{x} \cap A C_{r e f}\right)}{A C_{X} \cup A C_{r e f}}
$$

where $A C_{x}$ is a given attenuation map. The mean and standard deviation values across all participants were calculated.

Additionally, for the comparison between $\mathrm{AC}_{\mathrm{CT}}$ and $\mathrm{AC}_{\mathrm{ref}}$, difference (Diff) and absolute difference (AbsDiff) maps were calculated for all subjects. The MNI $2 \mathrm{~mm}$ head template was non-rigidly registered to the subject space. The above coefficients were calculated within the head to eliminate the impact from the background.

\section{PET reconstructed images comparison}

To evaluate PET reconstructed images with respect to $\mathrm{PET}_{\text {ref }}$ (PET images reconstructed with $\mathrm{AC}_{\text {ref }}$ attenuation maps), voxel-wise \%-difference maps were calculated (Eq. 2.).

$$
R=100 \cdot \frac{P E T_{r e f}-P E T_{X}}{P E T_{r e f}}
$$

where $P E T_{X}$ stands for PET images reconstructed with one of the evaluated attenuation coefficients maps.

\section{Analysis of the test participant}

In order to determine the accuracy of bone and air segmentation using the proposed method, the participant who undertook the PET examination, but had not been used to generate the template (referred to as the Test Participant), was also manually segmented into three tissue types: bone, soft tissue, and air. Segmentation was performed under the supervision of the clinical radiologist (NF). The quantitative accuracy of the reconstructed PET images for the Test Participant was assessed, and the normalised relative error across the whole brain was calculated.

\section{Whole brain PET performance}

To evaluate global PET performance whole brain masks for all participants were created. The 2-mm MNI atlas was aligned to each participant's PET image using linear and non-linear registration with T1w-MPRAGE images. Inverse transformed brain masks were used to calculate voxel-wise \%-difference with respect to $\mathrm{PET}_{\text {ref }}$ for all participants, defined in $E q .2$.

Additionally, the most representative histograms for the brain region were in order to visualise the trend of the differences between the techniques. For all subjects and reconstructed images, we produced histograms with the fixed number of bins (150). The bin width was fixed among methods but varied across subjects due to different uptake level as PET images were not intensity normalized. The $5 \times 150$ (no. of subjects $x$ no. of bins) matrix were then decomposed using SVD (Singular Value Decomposition) and the most representative histograms were calculated.

\section{Regional PET performance}

Fifteen predefined regions from the MNI space were inversely transformed to the subjects' spaces, and the relative error for each participant was calculated. For group analysis, the mean relative error across all participants was calculated. The average and standard deviation volumes of the $R E$ (Relative Error) were also calculated. Additionally, Mann-Whitney $U$-tests were performed between the different AC methods. A $p$ value of lower than 0.05 was considered statistically significant. Statistical comparisons 
were not investigated between the $\mathrm{PET}_{\mathrm{sUTEfix}}$ and PET $_{\text {sUTEcont }}$ methods.

\section{Effect of AC values for brain soft tissues}

To investigate the impact of the different attenuation coefficient assignments for WM, GM and CSF, one additional attenuation coefficient map was produced for the PET group. The following values: $0.151 \mathrm{~cm}^{-1}, 0.1 \mathrm{~cm}^{-1}$, $0.099 \mathrm{~cm}^{-1}, 0.099 \mathrm{~cm}^{-1}, 0.096 \mathrm{~cm}^{-1}$ and $0 \mathrm{~cm}^{-1}$ were assigned for bone, soft non-brain tissue, GM, WM, CSF and air, respectively. The resulting $\mathrm{AC}$ maps were smoothed with a 2-mm kernel Gaussian filter $\left(\mathrm{AC}_{\mathrm{mcAC}}\right)$.

As in the previous studies, the voxel-wise \%-difference between $\mathrm{PET}_{\text {ref }}$ and $\mathrm{PET}_{\mathrm{mcAC}}$ were computed with the $\mathrm{PET}_{\text {ref }}$ used as the reference, and regional \%-differences were also investigated.

\section{Results}

Comparisons of classification accuracy in air and bone in CT group

Comparison of $\mathrm{AC}_{\mathrm{ref}}$ and $\mathrm{AC}_{\mathrm{CT}}$ maps are shown in Fig. 3. Visual comparison shows very good agreement between the $\mathrm{AC}_{\text {ref }}$ and $\mathrm{AC}_{\mathrm{CT}}$. Co-localization of bone and air within the brain is excellent. The differences are seen in skull bones thickness, where $\mathrm{AC}_{\mathrm{CT}}$ has tendency to overestimate bones, and in sinuses, where the CT and MR co-registration shows large error.

Quantitative comparison between the attenuation maps across all subjects within the CT group is shown in Tables 3 and 4. Table 3 shows the DICE, FP and FN rates within the three tissue classes, i.e. air, soft tissue and bones. Averaged DICE is also calculated. Excellent agreement for air and soft tissue are shown and high similarity for bones between the two maps. The mean $\mathrm{FP}$ rate for bones $(\mathrm{FP}=0.219)$ is relatively high, suggesting overestimation of bones in $\mathrm{AC}_{\mathrm{CT}}$ and this is confirmed by visual inspection in the skull region. Averaged DICE show very good consistency between both methods with the mean value equal 0.917 . Table 4 reveals very low mean differences across all subjects between the compared techniques resulted in $0.0004 \mathrm{~cm}^{-1}$ and $0.0064 \mathrm{~cm}^{-1}$ values in relative difference and absolute difference, respectively.

\section{Comparisons of classification accuracy in air and bone in the PET group}

The attenuation correction maps for the different methods for the Test Participant are shown in Fig. 4. The proposed sUTE method shows improved classification of nasal cavities, temporal bones, and regions around the teeth, when compared with the conventional $\mathrm{AC}_{\mathrm{UTE}}$ method. The

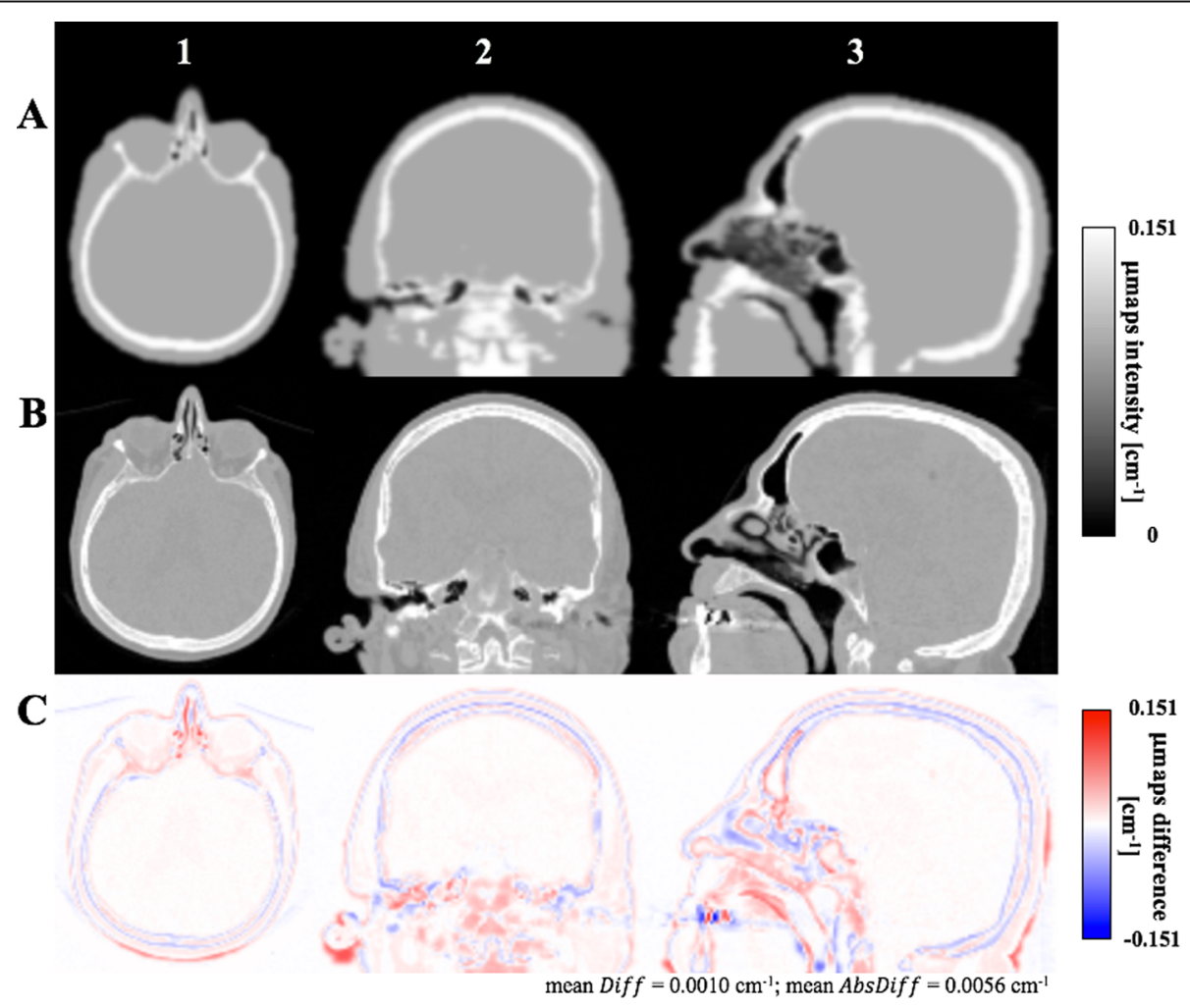

Fig. 3 Comparison of attenuation maps from $C T$ group. Comparison of attenuation maps for $A C_{r e f}$ (panel a) and $A C_{C T}$ (panel $\mathbf{b}$ ) in axial (column 1), sagittal (column 2) and coronal (column 3) view for the subject no. 4 from $C T$ group. Relative error between $A C_{\text {ref }}$ and $A C_{C T}$ is depicted in panel c 
Table 3 Different accuracy matrices for the CT-based segmentation method within the MNI space mask

\begin{tabular}{|c|c|c|c|c|c|c|c|c|c|c|}
\hline \multirow[t]{2}{*}{ Subject no. } & \multicolumn{3}{|l|}{ Air } & \multicolumn{3}{|c|}{ Soft Tissue } & \multicolumn{3}{|l|}{ Bones } & \multirow[t]{2}{*}{ Averaged DICE } \\
\hline & $\overline{D I C E}$ & $\mathrm{FP}$ & $\mathrm{FN}$ & $\overline{D I C E}$ & FP & FN & DICE & $\mathrm{FP}$ & $\mathrm{FN}$ & \\
\hline 01 & 0.974 & 0.033 & 0.017 & 0.933 & 0.055 & 0.060 & 0.792 & 0.226 & 0.019 & 0.900 \\
\hline 02 & 0.991 & 0.010 & 0.008 & 0.962 & 0.028 & 0.039 & 0.831 & 0.211 & 0.010 & 0.928 \\
\hline 03 & 0.983 & 0.024 & 0.009 & 0.955 & 0.030 & 0.052 & 0.837 & 0.203 & 0.010 & 0.925 \\
\hline 04 & 0.988 & 0.014 & 0.008 & 0.956 & 0.034 & 0.047 & 0.797 & 0.237 & 0.014 & 0.914 \\
\hline Mean & 0.984 & 0.020 & 0.010 & 0.952 & 0.037 & 0.049 & 0.815 & 0.219 & 0.014 & 0.917 \\
\hline Std & 0.007 & 0.010 & 0.004 & 0.012 & 0.012 & 0.009 & 0.023 & 0.015 & 0.004 & 0.013 \\
\hline
\end{tabular}

(Air threshold $=-500 \mathrm{HU}$; bone threshold $=300 \mathrm{HU}$ for $\mathrm{CT}$ images)

cortical bones are more accurately classified with the proposed method, compared to $\mathrm{AC}_{\mathrm{UCL}}$ which shows an overestimation of cortical bones. Bones in nasal cavities are slightly overestimated in the proposed method, whereas both the $\mathrm{AC}_{\mathrm{UCL}}$ and $\mathrm{AC}_{\mathrm{UTE}}$ methods underestimate bones, but overestimate the size of air cavity in this region.

In Table 5, the proposed method shows excellent accuracy in segmenting air and tissue with a DICE coefficient of 0.985 . There were very low numbers of false positive mis-segmented air voxels in the top of the head $(\mathrm{FP}=0.007)$ compared to the other methods. The overestimation of the air tissue shows poorer FN coefficients ( $F N=0.022)$ for the proposed method compared to the $\mathrm{AC}_{\mathrm{UCL}}$ method $(\mathrm{FN}=0.018)$. The bone segmentation results for the $\mathrm{AC}_{\mathrm{UCL}}$ and the proposed method show improvements compared to the $\mathrm{AC}_{\mathrm{UTE}}$ technique. Similar results are observed for false-positive and false-negative analyses across the evaluated methods, with the vendor's method showing the best FP result and the $\mathrm{AC}_{\mathrm{UCL}}$ method showing the best FN result. The proposed method, and the $\mathrm{AC}_{\mathrm{UCL}}$, method, show better segmentation compared to the $\mathrm{AC}_{\mathrm{UTE}}$ technique.

\section{Comparison of PET images}

The reconstructed PET images of the Test Participant using different attenuation correction maps, and the normalized error maps, are shown in Fig. 5. Comparing the reconstructed $\mathrm{PET}$ images, the proposed $\mathrm{PET}_{\mathrm{sUTEfix}}$

Table 4 Mean difference (Diff) and mean absolute difference (AbsDiff) between manual segmentation based attenuation maps and $\mathrm{CT}$ based attenuation maps for each subject from CT group

\begin{tabular}{llllll}
\hline Subject no. & \multicolumn{2}{l}{ Diff $\left[\mathrm{cm}^{-1}\right]$} & & \multicolumn{2}{l}{ AbsDiff $\left[\mathrm{cm}^{-1}\right]$} \\
\cline { 2 - 3 } & Mean & SD & & Mean & SD \\
\hline 01 & 0.0013 & 0.0145 & & 0.0072 & 0.0126 \\
02 & -0.0004 & 0.0110 & & 0.0059 & 0.0093 \\
03 & -0.0002 & 0.0127 & & 0.0068 & 0.0108 \\
04 & 0.0010 & 0.0113 & & 0.0056 & 0.0098 \\
Mean & 0.0004 & & & 0.0064 & \\
\hline
\end{tabular}

and $\mathrm{PET}_{\mathrm{suTEcont}}$ show the overall most accurate reconstruction with the lowest normalized reconstruction error. The $\mathrm{PET}_{\mathrm{UTE}}$ images show a significant underestimation of activity in the whole brain. On the other hand, the $\mathrm{PET}_{\mathrm{UCL}}$ method shows an increased uptake at tissues adjacent to the cortical bones, especially in the parietal lobe grey matter, up to $20 \%$, and in the brain stem, lateral ventricle and thalamus, where underestimated activity uptake is observed. The proposed methods show reconstruction errors around the cortex due to errors in the cortical bone estimation in the corresponding AC maps. Compared between the two proposed methods, the PET $\mathrm{PUTE}_{\mathrm{sU}}$ cont shows overall lower error due to a more accurate bone attenuation coefficient from the R2 ${ }^{*}$-based approaches.

\section{Comparison of group PET images}

Figure 6 shows the histograms of PET images for all participants in the cohort. The $\mathrm{PET}_{\mathrm{UTE}}$ shows a lower mean uptake compared with other methods. The $\mathrm{PET}_{\mathrm{UCL}}$ histogram shows a wider spread of uptake values in comparison with the other methods. Compared with the reference $\mathrm{PET}_{\text {ref }}$ images, both the PET $\mathrm{T}_{\text {sUTEfix }}$ and $\mathrm{PET}_{\text {sUTEcont }}$ show similar intensity distributions, with the $\mathrm{PET}_{\text {sUTEfix }}$ image demonstrating slightly more accurate results compared to the $\mathrm{PET}_{\text {sUTEcont }}$ image.

\section{Comparison of PET performance in brain regions}

The mean $R E \%$ of the whole brain, and across the brain regions, is shown in Fig. 7. The PET images reconstructed with the proposed attenuation correction method show excellent agreement with the reference $\mathrm{PET}_{\text {ref }}$ images. The most significant differences are observed in the cerebellum, where the $\mathrm{PET}_{\mathrm{UCL}}, \mathrm{PET}_{\mathrm{sUTE}}$ ant and $\mathrm{PET}_{\mathrm{SUTEfix}}$ images overestimate the PET signal by $4.6,3.4$ and $2.8 \%$, respectively, whereas the $\mathrm{PET}_{\mathrm{UTE}}$ image gives a significantly lower uptake $(-6.4 \%)$. The $\mathrm{PET}_{\mathrm{UCL}}$ method generally overestimates the activity for all brain regions, with a mean of 3.8\% for the whole brain. Both the $\mathrm{PET}_{\text {sUTEcont }}$ and $\mathrm{PET}_{\text {sUTEfix }}$ images have very similar full brain activity uptake across the subjects compared with the reference. Detailed results are presented in Table 6 . 


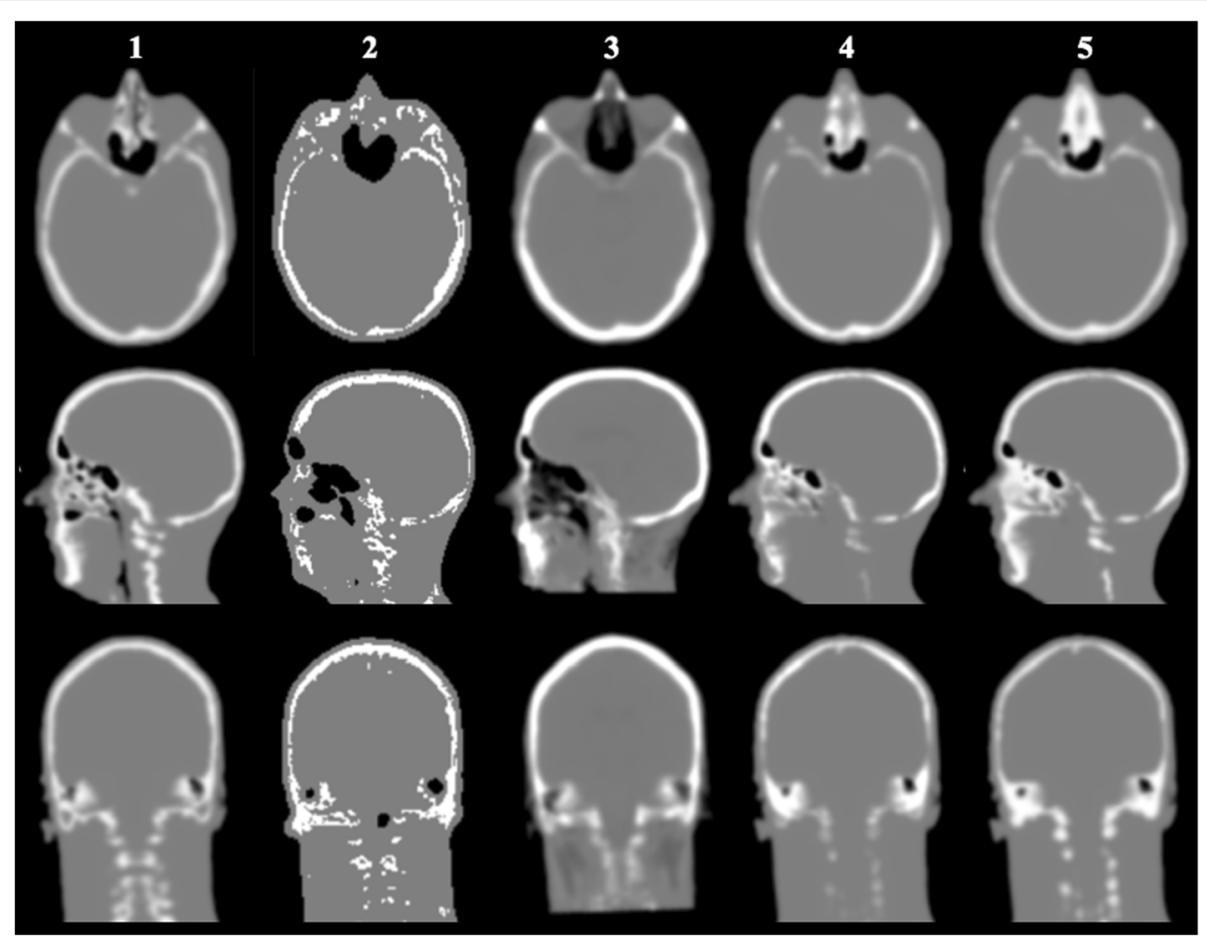

Fig. 4 Comparison of attenuation correction maps for the Test participant. Comparison of attenuation correction maps for $A C_{\text {ref }}$ (column 1), $A C_{\text {UTE }}$ (column 2), $A C_{\text {UCL }}$ (column 3), $A C_{\text {sUTEcont }}$ (column 4) and $\mathrm{AC}_{\text {sUTEfix }}$ (column 5) in axial (top), sagittal (centre) and coronal (bottom) view for the Test participant

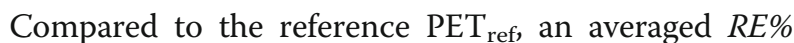
image across all subjects is obtained, as in Fig. 8, and the standard deviation image is shown in Fig. 9. The group $R E \%$ image (Fig. 8) confirms the superiority of both the $\mathrm{PET}_{\mathrm{sUTEfix}}$ and $\mathrm{PET}_{\mathrm{sUTE}}$ ment methods.

\section{Evaluation of multi-compartment soft tissue AC map}

To investigate the impact of multi-compartment AC coefficients in soft tissue, $\mathrm{PET}_{\mathrm{mcAC}}$ is reconstructed using multi-compartment soft tissue models. The mean RE\% between the $\mathrm{PET}_{\text {ref }}$ and $\mathrm{PET}_{\text {mcAC }}$ images across several anatomical regions and the whole brain is shown in Fig. 10. The reduction of activity estimation is between 1 and $3 \%$ for a number of brain regions. The most significant differences are seen in the regions near the ventricles: caudate, hippocampus, thalamus, etc. The average $R E \%$ and standard deviation images are shown in Fig. 11.

\section{Discussion}

This paper introduces a new PET AC map estimation method for simultaneous PET/MR imaging. Unlike conventional MR-CT atlas based methods, e.g. $\mathrm{AC}_{\mathrm{UCL}}$, the new method is fully based on MR data. We have created a bone/air/soft tissue template based on the manual segmentation of UTE images using data from five participants. Gaussian mixture models are then used to fit UTE images to generate new AC maps. We show that manual segmentation based attenuation correction maps are comparable to the $\mathrm{CT}$ based attenuation maps. Compared with the reference AC map, the proposed sUTE method shows very good results for the whole brain region with the DICE coefficient equal to 0.985 and 0.737 for air and bone, respectively. The proposed method shows significant improvements in reduction of the reconstruction errors, compared with the $\mathrm{AC}_{\mathrm{UTE}}$ and $\mathrm{AC}_{\mathrm{UCL}}$ methods.

Table 5 Different accuracy matrices for the different segmentation methods for the PET group

\begin{tabular}{|c|c|c|c|c|c|c|}
\hline \multirow[t]{2}{*}{ Method } & \multicolumn{3}{|l|}{ Air } & \multicolumn{3}{|l|}{ Bones } \\
\hline & DICE & FP & FN & DICE & FP & $\mathrm{FN}$ \\
\hline UTE & $0.953(006)$ & $0.091(015)$ & $0.006(003)$ & $0.641(042)$ & $0.204(028)$ & $0.431(043)$ \\
\hline sUTE & $0.985(002)$ & $0.007(007)$ & $0.022(009)$ & $0.737(017)$ & $0.215(084)$ & $0.277(098)$ \\
\hline UCL & $0.971(024)$ & $0.041(062)$ & $0.018(011)$ & $0.780(024)$ & $0.371(034)$ & $0.123(023)$ \\
\hline
\end{tabular}

The best results are shown in bold 


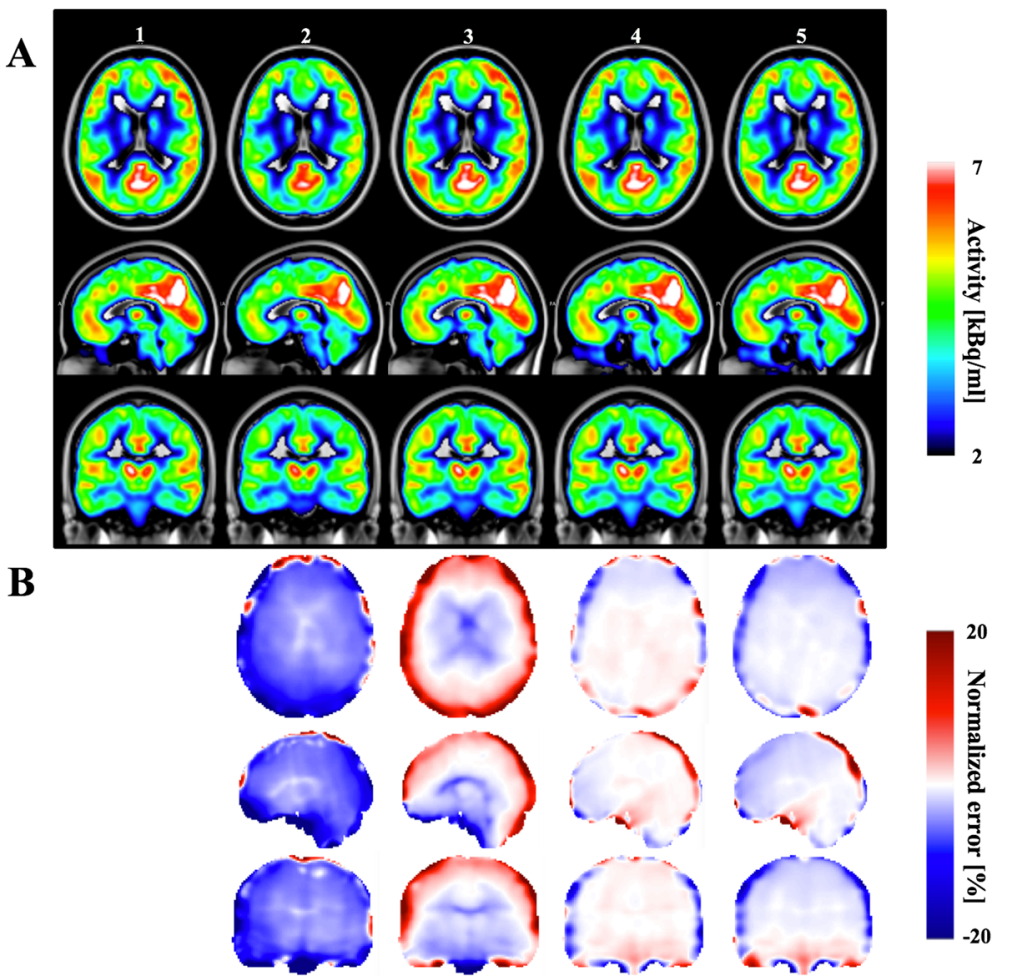

Fig. 5 Comparison of PET images for the Test participant. Comparison of PET images for the Test participant (panel a) reconstructed with five

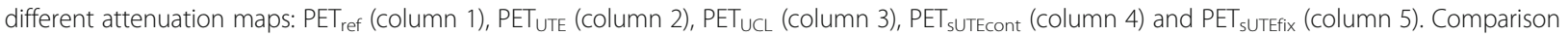
of corresponding normalized error (panel b) for the reconstructed PET data is depicted

The proposed $\mathrm{AC}_{\text {sUTEfix }}$ and $\mathrm{AC}_{\text {sUTEcont }}$ maps show excellent agreement with the reference AC map (Fig. 4), especially in the region of the cortical bones, where the $\mathrm{AC}_{\mathrm{UCL}}$ shows an overestimation of the $\mathrm{AC}$ coefficients. These differences in AC maps are also confirmed by the false positive and false negative scores in Table 5 .
Differences in the DICE coefficients, false-negative and false-positive scores, demonstrate the difficulties with bone and air segmentation. In terms of the classification of air cavities in the head and neck, the best results are obtained using the proposed method, albeit with a slight underestimation in the oesophagus. Very low false

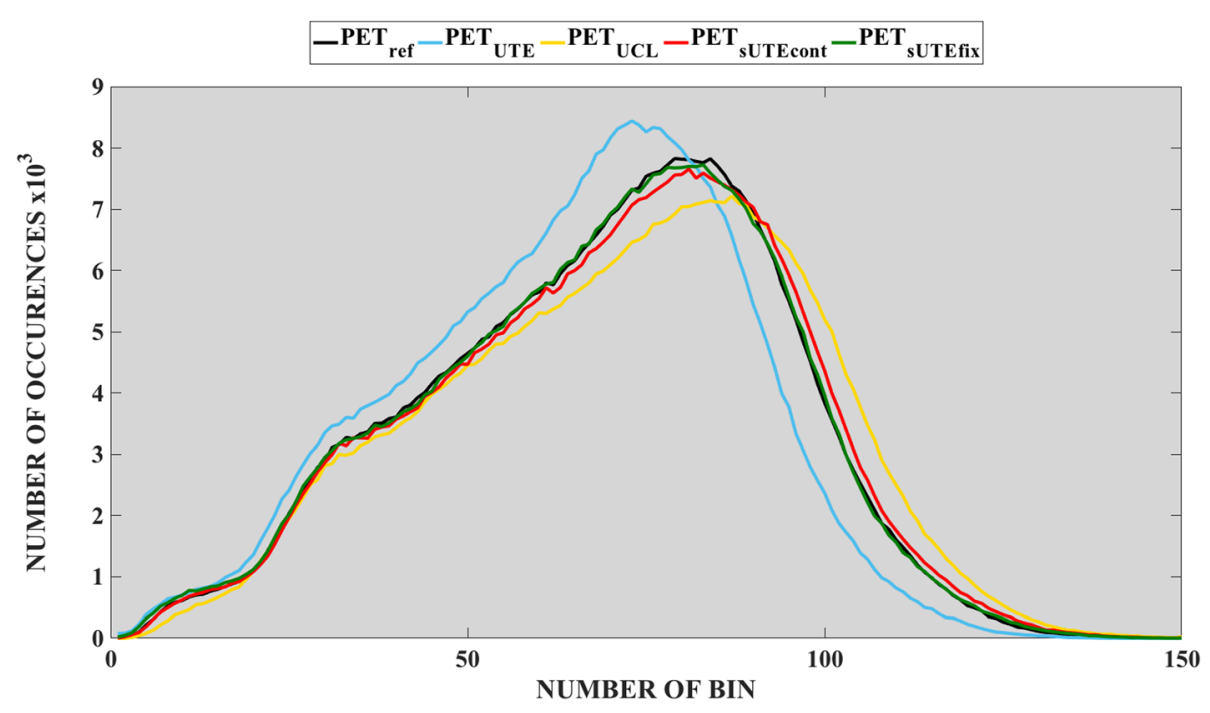

Fig. 6 Histograms of the reconstructed PET images. Histograms of the reconstructed PET images within the brain for the group using different methods 


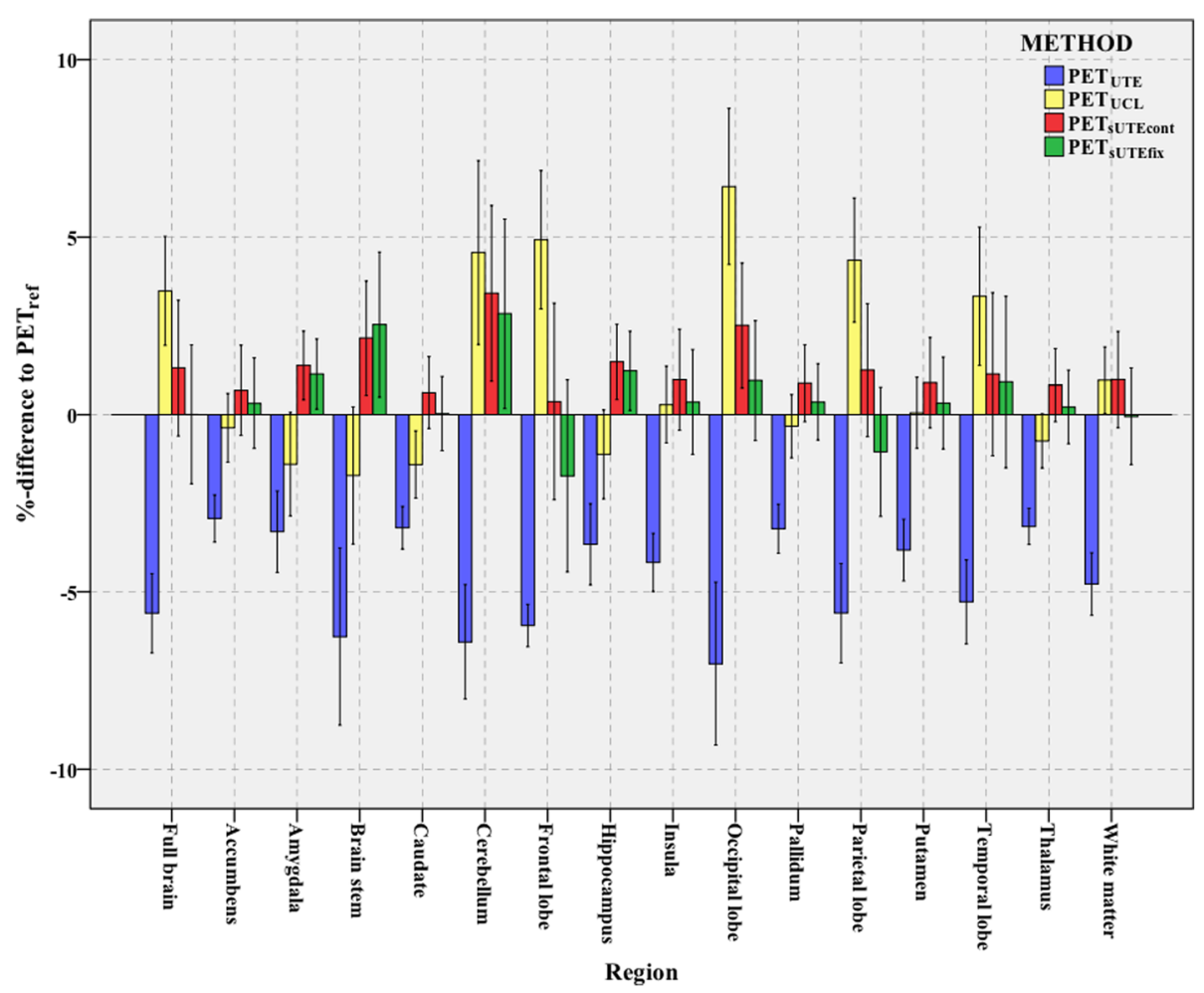

Fig. 7 Comparison of quantitative PET measurements. Comparison of quantitative PET measurements in various brain regions with respect to the reference (\%-difference \pm SD)

Table $6 \mathrm{ROI}$ analysis for the PET group

\begin{tabular}{|c|c|c|c|c|c|c|c|c|}
\hline \multirow[t]{3}{*}{ Region } & \multicolumn{8}{|c|}{ Method } \\
\hline & \multicolumn{2}{|c|}{ PET UTE $_{\text {- }}$ PET $_{\text {ref }}$} & \multicolumn{2}{|c|}{$P E T_{U C L}-P E T_{\text {ref }}$} & \multicolumn{2}{|c|}{$\mathrm{PET}_{\text {sUTEcont }}-\mathrm{PET}_{\text {ref }}$} & \multicolumn{2}{|c|}{$\mathrm{PET}_{\text {sUTEfix }}-P E T_{\text {ref }}$} \\
\hline & Mean & SD & Mean & SD & Mean & SD & Mean & SD \\
\hline Full brain & $-5.6^{*}$ & 1.1 & $3.8^{*}$ & 1.5 & 1.3 & 1.9 & 0.0 & 2.0 \\
\hline Accumbens & $-2.93^{*}$ & 0.67 & -0.37 & 0.97 & 0.7 & 1.3 & 0.3 & 1.3 \\
\hline Amygdala & $-3.3^{*}$ & 1.2 & -1.4 & 1.5 & $1.38^{*}$ & 0.97 & $1.14^{*}$ & 0.98 \\
\hline Brain stem & $-6.3^{*}$ & 2.5 & $-1.7^{*}$ & 1.9 & $2.2^{*}$ & 1.6 & $2.5^{*}$ & 2.0 \\
\hline Caudate & $-3.20^{*}$ & 0.60 & -1.41 & 0.95 & 0.6 & 1.0 & 0.0 & 1.0 \\
\hline Cerebellum & $-6.4^{*}$ & 1.6 & $4.6^{*}$ & 2.6 & $3.4^{*}$ & 2.5 & 2.8 & 2.7 \\
\hline Frontal lobe & $-5.95^{*}$ & 0.59 & $4.9^{*}$ & 2.0 & 0.4 & 2.8 & -1.7 & 2.7 \\
\hline Hippocampus & $-3.7^{*}$ & 1.2 & $-1.1^{*}$ & 1.3 & $1.5^{*}$ & 1.1 & $1.2^{*}$ & 1.1 \\
\hline Insula & $-4.17^{*}$ & 0.82 & 0.3 & 1.1 & 1.0 & 1.4 & 0.4 & 1.5 \\
\hline Occipital lobe & $-7.0^{*}$ & 2.3 & $6.4^{*}$ & 2.2 & $2.5^{*}$ & 1.8 & 1.0 & 1.7 \\
\hline Pallidum & $-3.22^{*}$ & 0.69 & -0.33 & 0.89 & 0.9 & 1.1 & 0.4 & 1.1 \\
\hline Parietal lobe & $-5.6^{*}$ & 1.4 & $4.4^{*}$ & 1.8 & 1.3 & 1.9 & -1.1 & 1.8 \\
\hline Putamen & $-3.82^{*}$ & 0.87 & 0.1 & 1.0 & 0.9 & 1.3 & 0.3 & 1.3 \\
\hline Temporal lobe & $-5.3^{*}$ & 1.2 & 3.33 & 2.0 & 1.1 & 2.3 & 0.9 & 2.4 \\
\hline Thalamus & $-3.16^{*}$ & 0.51 & -0.74 & 0.77 & 0.8 & 1.0 & 0.2 & 1.0 \\
\hline White matter & $-4.78^{*}$ & 0.88 & 0.96 & 0.94 & 1.0 & 1.4 & -0.0 & 1.4 \\
\hline
\end{tabular}

${ }^{*}$ - differences between tested method and $\mathrm{PET}_{\text {ref }}$ considered statistically significant $(p<0.05)$ 


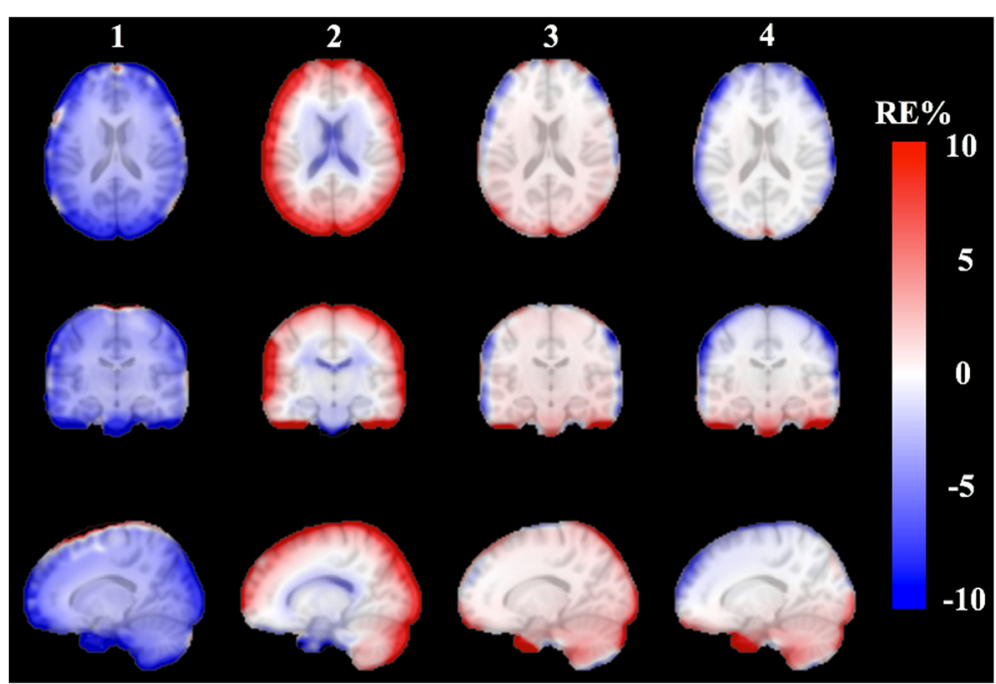

Fig. 8 Group averaged RE\% images in the MNI template space. Group averaged RE\% images in the MNI template space across all subjects for each method: (1) PET UTE, (2) PETUCL, (3) PET sUTEcont and (4) PET SUTEfix

negative scores for the $\mathrm{AC}_{\mathrm{UTE}}$ method in the surrounding air are likely to result from significant underestimation of air cavities. The improved classification of air in the new method is due to the excellent air/soft-tissue contrast in the MR UTE template, compared with that in the CT template.

Overall and regional PET performance analyses demonstrate good agreement of the $\mathrm{PET}_{\text {SUTEfix }}$ and $\mathrm{PET}_{\text {sUTEcont }}$ images with the reference image. The histograms of $\mathrm{PET}_{\text {ref }}$ and $\mathrm{PET}_{\text {sUTEfix }}$ in the group study are the most similar. In the averaged $R E \%$ images, the overall higher uptake in grey matter adjacent to cortical bones in $\mathrm{PET}_{\mathrm{UCL}}$ is caused by bone region overestimation and atlas-based attenuation coefficient values which are greater than those used in other methods (particularly in the occipital cortex). Furthermore, the cerebellum is a critical region for proper PET quantitative assessment and kinetic modeling, as it is used as a reference region. The $\mathrm{PET}_{\text {sUTEcont }}$ and $\mathrm{PET}_{\text {sUTEfix }}$ images underestimate regions in the frontal pole and frontal gyrus, and overestimate the signal in the postcentral gyrus and the brain stem. All of the methods show varying levels of error compared to the $\mathrm{PET}_{\text {ref }}$ image in the above-mentioned areas, due to difficulties of accurate classification in the nasal cavities, the base of the skull and occipital bone, which are adjacent to important areas for ${ }^{18}$ F-FDG PET quantification.

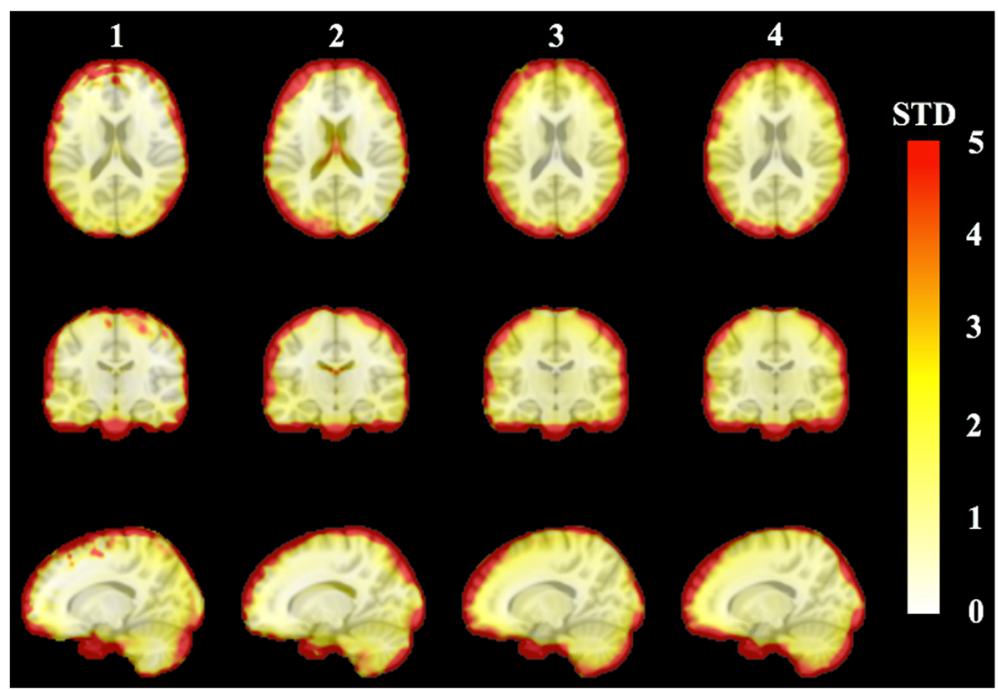

Fig. 9 Group standard deviation images in the MNI template space. Group standard deviation images in the MNI template space across all subjects for each method: (1) PET UTE, (2) PET UCL, (3) PET sUTEcont, and (4) PET $_{\text {sUTEfix }}$ 


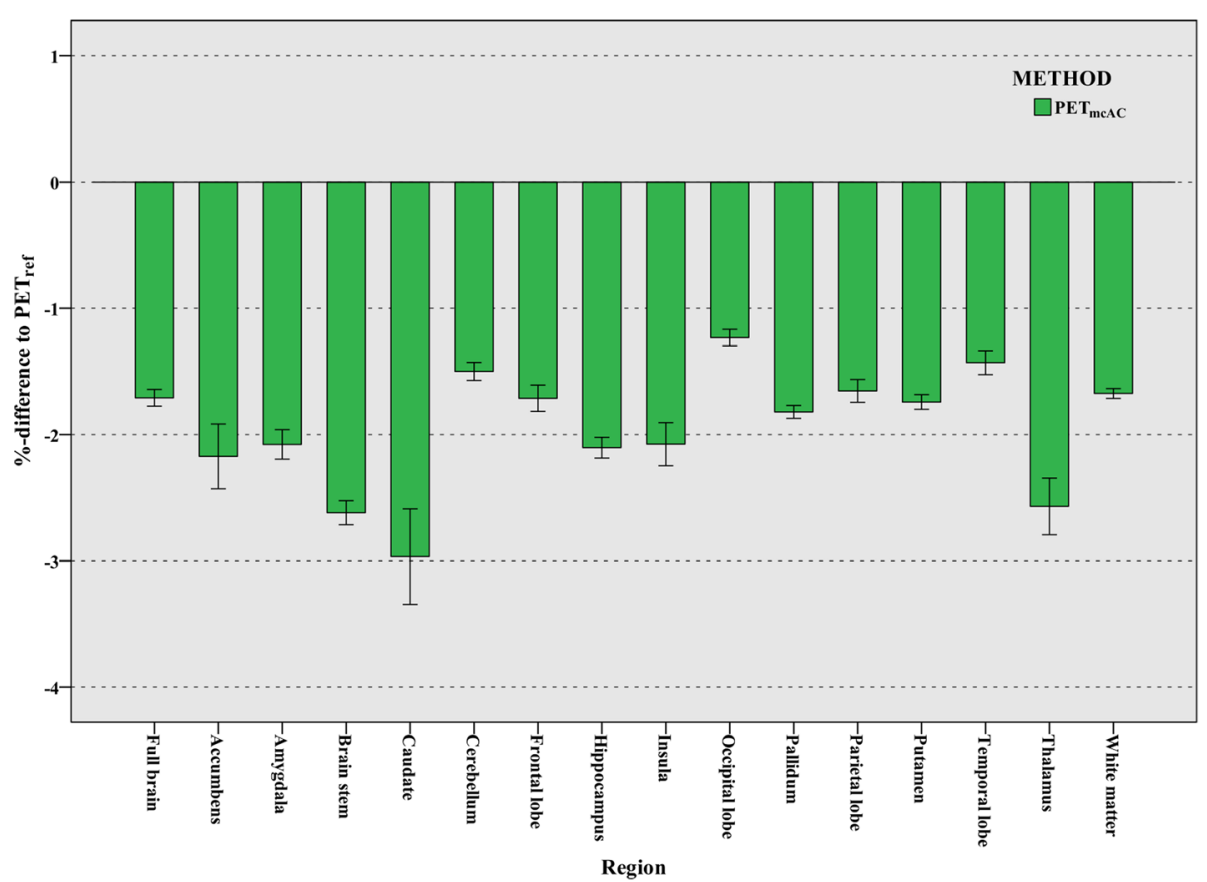

Fig. 10 Comparison of reconstructed images. Comparison of reconstructed images using 3-compartment soft tissue AC (PET mcAC) and 1compartment soft tissue AC (PET ref $)$

We compared the group variability for the three different methods, with all methods demonstrating some variability in the cortical areas, compared to the reference image. This indicates the importance of accurate bone segmentation and the necessity of subject-specific linear attenuation coefficients, especially considering that cortical bone density can vary systematically between individuals. Towards this end, the UTE R2* based method is potentially more accurate, as it measures subject-specific information, whereas atlas-based methods are limited by the richness of their atlas and tend to regress every subject towards the mean.
The selection of a reference AC map for the PET/MR is not straightforward. Due to absence of gold standard transmission scans, the CT based attenuation maps are widely used as a silver standard for that purpose. However, very early study on the PET attenuation correction [57] showed statistically significant differences between transmission based and CT based attenuation maps and resulted in the overestimation of the PET uptake results using $\mathrm{CT}$ attenuation maps. As a one of the major issues, Nakomoto et al. indicated the problem of the conversion from $\mathrm{HU}$ to $511 \mathrm{keV}$ attenuation correction factors. Furthermore, registration errors between $\mathrm{CT}$ and MR

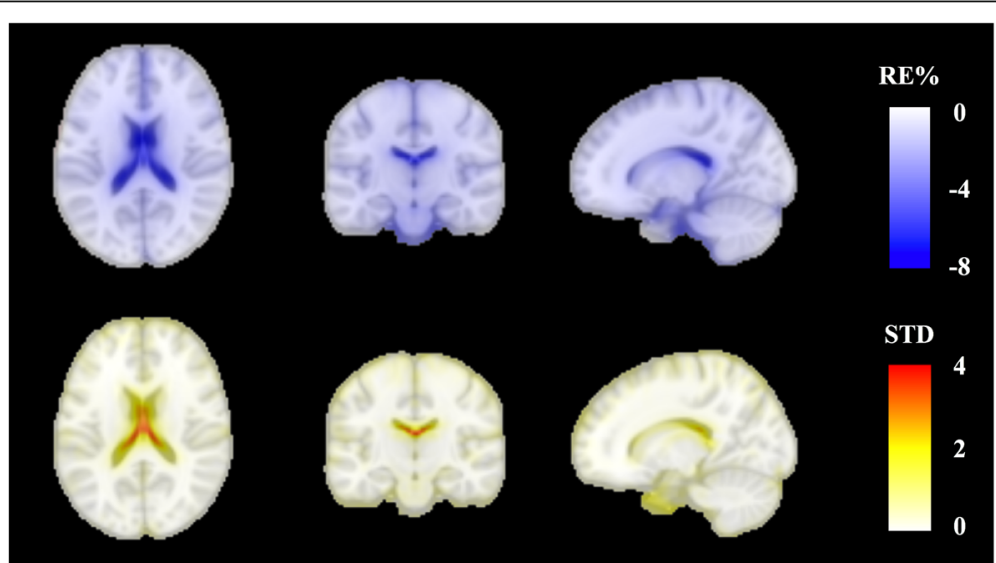

Fig. 11 Group averaged RE\% and standard deviation images in the MNI space. Group averaged RE\% (top row) and standard deviation (bottom row) images in the MNI space across all subjects for the 3-compartment soft tissue model with respect to 1-compartment soft tissue model 
can potentially be a source of error in PET/MR attenuation correction as discussed in [53]. In this paper, we have evaluated an alternative way of creating reference AC maps based on anatomical delineation of tissue classes.

Due to the lack of knowledge of the true attenuation coefficients, we have investigated the impact of the number of compartments used for soft tissue attenuation correction. The overall difference is around 2\%, compared with the 1- and 3-compartment brain tissue models, where the CSF and brainstem show the greatest differences due to the lower CSF attenuation factor of $0.096 \mathrm{~cm}^{-1}$ in the 3-compartment model.

\section{Limitations and future work}

There are several limitations to this study. The first limitation is the absence of an absolute reference for the attenuation coefficient maps to compare results from different methods. The absolute ground truth reference for PET attenuation correction studies is measured using a $511 \mathrm{keV}$ transmission scan in the PET acquisition [57]. Other studies have used CT scans to measure and compute a reference AC map for $511 \mathrm{keV}$. However, standard CT images correspond to linear attenuation coefficients for photons with an energy range between 80 and $140 \mathrm{keV}$, with the extrapolation from the CT to PET attenuation coefficient values potentially introducing a systematic bias. The problem with having an absolute reference might be addressed by TOF MLAA methods which could generate attenuation correction maps estimated from the PET-scanner emission data.

In this study, the reference AC map is generated using manual segmentation of brain tissue, bones and air cavities according to the anatomical landmarks used in standard clinical practice. Similar to other template based methods, there may be potential segmentation and registration errors. However, compared with the templates created from other modalities, the sUTE methods are potentially more accurate to the excellent soft tissue contrast from MRI.

Furthermore, this study includes a small number of healthy participants and focuses on a particular age group. In future work, we plan to investigate the effects of age differences (i.e. bone density differences with ageing) and investigate the attenuation coefficient differences using both PET/CT and PET/MR scanning in healthy and disease groups.

\section{Conclusion}

We propose a novel attenuation correction method for PET data reconstruction, with a common segmentation step, but different approaches for assignment of the bone attenuation coefficients. The first method uses a fixed attenuation coefficient for bone, whereas the second method employs R2* map conversion to determine the PET attenuation coefficients. Visual and quantitative analyses show significant improvements in the quantitative accuracy of the reconstructed PET images compared to standard methods, and improved results compared to other state-of-the-art AC methods for simultaneous PET/MR scanners. Accurate air segmentation is a significant advantage of the proposed method, and potentially enables the reconstructed PET images to be used for quantitative neurological brain imaging. However, further evaluation is needed with radiopharmaceuticals other than FDG, and in larger cohorts of participants, including those with neurological diseases and focal lesions.

\section{Abbreviations \\ AC: Attenuation coefficients maps; CSF: Cerebrospinal fluid; CT: Computed tomography; dUTE: Dual ultrashort echo time sequence; FDG: Fluorodeoxyglucose; FN: False-negative; FOV: Field-of-view; FP: False-positive; GM: Grey matter; HU: Hounsfield Units; MLAA: Maximum likelihood attenuation and activity reconstruction algorithm; MNI: Montreal Neurological Institute; MR: Magnetic resonance; OP-OSEM: Ordinary Poisson - Ordered Subset Expectation Maximization algorithm; PET: Positron Emission Tomography; PET/MR: Positron Emission Tomography and Magnetic Resonance; PSF: Point Spread Function; RE: Relative error; SPM12: Statistical parametric mapping software; sUTE: Segmented UTE; SVD: Singular Value Decomposition; TE1: First echo time; TE2: Second echo time; TOF: Time-of-flight; TPM: Tissue probability maps; TR: Repetition time; UTE1: First echo UTE image; UTE2: Second echo UTE image; WM: White matter}

\section{Acknowledgments}

We acknowledge Siemens Healthineers Australia for their help in facilitating this research. Jakub Baran would like to acknowledge the grant received from Monash University that allowed him to work at Monash Biomedical Imaging. We acknowledge Dr. Elena Rota-Kops from Institute of Neuroscience and Medicine, Forschungszentrum Juelich $\mathrm{GmbH}$, Juelich, Germany for sharing the CT and MRI datasets for this study.

\section{Funding}

The experiments in this study were supported by a grant from the Reignwood Cultural Foundation and by an Australian Research Council (ARC) Discovery Early Career Researcher fellowship (DE150100406). The analysis work was supported by the Reignwood Cultural Foundation grant and by an ARC grant CE1400007.

\section{Availability of data and materials}

The raw datasets used during the current study can be made available from the corresponding author on reasonable request pending the approval of the institution and the study investigators who contributed to the datasets.

\section{Authors' contributions}

All authors significantly contributed to the conception and design of the study. JB prepared the participant data, interpreted the results and drafted the manuscript. JB and FS performed the analyses, and NJS, DF and BS provided technical advice for the acquisition and post-processing methods. $J B, Z C, S J, M C$ and GE interpreted the data, and ZC, BS, DF, SJ, NF, MC, NJS and $G E$ revised the manuscript. Advice regarding the clinical aspects of the study were provided by NF. All authors contributed to the manuscript preparation and read and approved the final manuscript. Monash University owns the data.

\section{Ethics approval and consent to participate}

All examinations were approved by the Monash University Human Research Ethics Committee. The written consent forms were approved by the Monash University Human Research Ethics Committee and signed by the participants. 


\section{Competing interests}

The authors declare that they have no competing interests.

\section{Publisher's Note}

Springer Nature remains neutral with regard to jurisdictional claims in published maps and institutional affiliations.

\section{Author details}

'Monash Biomedical Imaging, Monash University, Melbourne, Australia. ${ }^{2}$ Department of Biophysics, Faculty of Mathematics and Natural Sciences, University of Rzeszow, Rzeszow, Poland. Institute of Nuclear Physics Polish Academy of Science, Krakow, Poland. ${ }^{4}$ Department of Electrical and Computer Systems Engineering, Monash University, Melbourne, Australia. ${ }^{5}$ Monash Imaging, Monash Health, Clayton, Australia. ${ }^{6}$ Monash Institute of Cognitive and Clinical Neurosciences and School of Psychological Sciences, Monash University, Melbourne, Australia. ${ }^{7}$ Australian Research Council Centre of Excellence for Integrative Brain Function, Monash University, Melbourne, Australia. ${ }^{8}$ Siemens Healthcare Pty Ltd, Sydney, Australia. ${ }^{9}$ Siemens Healthcare Pty Ltd, New York, USA. ${ }^{10}$ Institute of Neuroscience and Medicine, Forschungszentrum Juelich GmbH, Juelich, Germany.

\section{Received: 1 August 2018 Accepted: 24 October 2018}

Published online: 06 November 2018

\section{References}

1. Razek AAKA. Diffusion magnetic resonance imaging of chest tumors. Cancer Imaging. 2012;12:452.

2. Razek AAKA. Routine and advanced diffusion imaging modules of the salivary glands. Neuroimaging Clin N Am. 2018;28:245-54.

3. Sepahdari AR, Politi LS, Aakalu VK, Kim HJ, Razek AAKA. Diffusion-weighted imaging of orbital masses: multi-institutional data support a 2-ADC threshold model to categorize lesions as benign, malignant, or indeterminate. Am J Neuroradiol. 2013.

4. Heiss W-D. The potential of PET/MR for brain imaging. Eur J Nucl Med Mol Imaging. 2009;36:105-12.

5. Sotoudeh H, Sharma A, Fowler KJ, McConathy J, Dehdashti F. Clinical application of PET/MRI in oncology. J Magn Reson Imaging. 2016;44:265-76.

6. Torigian DA, Zaidi H, Kwee TC, Saboury B, Udupa JK, Cho Z-H, et al. PET/MR imaging: technical aspects and potential clinical applications. Radiology. 2013;267:26-44

7. Aiello M, Cavaliere C, Salvatore M. Hybrid PET/MR imaging and brain connectivity. Front Neurosci. 2016;10:64

8. Miller-Thomas MM, Benzinger TLS. Neurologic applications of PET/MR imaging. Magn Reson Imaging Clin. 2017;25:297-313.

9. Chen Z, Jamadar SD, Li S, Sforazzini F, Baran J, Ferris N, et al. From simultaneous to synergistic MR-PET brain imaging: a review of hybrid MRPET imaging methodologies. Hum Brain Mapp. 2018.

10. Burger C, Goerres G, Schoenes S, Buck A, Lonn A, Von Schulthess G. PET attenuation coefficients from $C T$ images: experimental evaluation of the transformation of CT into PET 511-keV attenuation coefficients. Eur J Nucl Med Mol Imaging. 2002;29:922-7.

11. Carney JPJ, Townsend DW, Rappoport V, Bendriem B. Method for transforming $C T$ images for attenuation correction in PET/CT imaging. Med Phys. 2006;33:976-83.

12. Kinahan PE, Townsend DW, Beyer T, Sashin D. Attenuation correction for a combined 3D PET/CT scanner. Med Phys. 1998;25:2046-53.

13. Andersen FL, Ladefoged CN, Beyer T, Keller SH, Hansen AE, Højgaard L, et al. Combined PET/MR imaging in neurology: MR-based attenuation correction implies a strong spatial bias when ignoring bone. Neurolmage. 2014;84: 206-16.

14. Akbarzadeh A, Ay MR, Ahmadian A, Alam NR, Zaidi H. MRl-guided attenuation correction in whole-body PET/MR: assessment of the effect of bone attenuation. Ann Nucl Med. 2013;27:152-62.

15. Martinez-Moller A, Souvatzoglou M, Delso G, Bundschuh RA, Chefd'hotel C, Ziegler SI, et al. Tissue classification as a potential approach for attenuation correction in whole-body PET/MRI: evaluation with PET/CT data. J Nucl Med. 2009;50:520

16. Dickson JC, O'Meara C, Barnes A. A comparison of CT-and MR-based attenuation correction in neurological PET. Eur J Nucl Med Mol Imaging. 2014:41:1176-89.
17. Koesters T, Friedman KP, Fenchel M, Zhan Y, Hermosillo G, Babb J, et al. Dixon sequence with superimposed model-based bone compartment provides highly accurate PET/MR attenuation correction of the brain. J Nucl Med Off Publ Soc Nucl Med. 2016:57:918.

18. Paulus DH, Quick HH, Geppert C, Fenchel M, Zhan Y, Hermosillo G, et al. Whole-body PET/MR imaging: quantitative evaluation of a novel modelbased MR attenuation correction method including bone. J Nucl Med Off Publ Soc Nucl Med. 2015;56:1061.

19. Wollenweber SD, Ambwani S, Lonn AHR, Shanbhag DD, Thiruvenkadam S, Kaushik S, et al. Comparison of 4-class and continuous fat/water methods for whole-body, MR-based PET attenuation correction. IEEE Trans Nucl Sci. 2013;60:3391-8.

20. Nuyts J, Dupont $P$, Stroobants S, Benninck R, Mortelmans L, Suetens P. Simultaneous maximum a posteriori reconstruction of attenuation and activity distributions from emission sinograms. IEEE Trans Med Imaging. 1999;18:393-403.

21. Mehranian A, Zaidi $\mathrm{H}$. Emission-based estimation of lung attenuation coefficients for attenuation correction in time-of-flight PET/MR. Phys Med Biol. 2015;60:4813.

22. Salomon A, Goedicke A, Schweizer B, Aach T, Schulz V. Simultaneous reconstruction of activity and attenuation for PET/MR. IEEE Trans Med Imaging. 2011;30:804-13.

23. Benoit D, Ladefoged CN, Rezaei A, Keller SH, Andersen FL, Højgaard L, et al. Optimized MLAA for quantitative non-TOF PET/MR of the brain. Phys Med Biol. 2016;61:8854

24. Rothfuss H, Panin V, Moor A, Young J, Hong I, Michel C, et al. LSO background radiation as a transmission source using time of flight. Phys Med Biol. 2014:59:5483.

25. Cabello J, Lukas M, Förster S, Pyka T, Nekolla SG, Ziegler SI. MR-based attenuation correction using ultrashort-echo-time pulse sequences in dementia patients. J Nucl Med. 2015;56:423-9.

26. Ladefoged CN, Benoit D, Law I, Holm S, Kjær A, Højgaard L, et al. Region specific optimization of continuous linear attenuation coefficients based on UTE (RESOLUTE): application to PET/MR brain imaging. Phys Med Biol. 2015;60:8047.

27. Catana C, van der Kouwe A, Benner T, Michel CJ, Hamm M, Fenchel M, et al. Towards implementing an MR-based PET attenuation correction method for neurological studies on the MR-PET brain prototype. J Nucl Med Off Publ Soc Nucl Med. 2010;51:1431.

28. Keereman V, Fierens $Y$, Broux T, De Deene $Y$, Lonneux M, Vandenberghe S. MRI-based attenuation correction for PET/MRI using ultrashort echo time sequences. J Nucl Med. 2010;51:812.

29. Juttukonda MR, Mersereau BG, Chen Y, Su Y, Rubin BG, Benzinger TLS, et al. MR-based attenuation correction for PET/MRI neurological studies with continuous-valued attenuation coefficients for bone through a conversion from R2* to CT-Hounsfield units. Neurolmage. 2015;112:160-8.

30. Ribeiro AS, Kops ER, Herzog H, Almeida P. Hybrid approach for attenuation correction in PET/MR scanners. Nucl Instruments Methods Phys Res Sect A Accel Spectrometers Detect Assoc Equip. 2014;734:166-70.

31. Leynes AP, Yang J, Wiesinger F, Kaushik SS, Shanbhag DD, Seo Y, et al. Direct pseudoCT generation for pelvis PET/MRI attenuation correction using deep convolutional neural networks with multi-parametric MRI: zero echotime and Dixon deep pseudoCT (ZeDD-CT). J Nucl Med. 2017;jnumed-117. http://jnm.snmjournals.org/content/early/2017/10/25/jnumed.117.198051

32. Leynes AP, Yang J, Shanbhag DD, Kaushik SS, Seo Y, Hope TA, et al. Hybrid ZTE/Dixon MR-based attenuation correction for quantitative uptake estimation of pelvic lesions in PET/MRI. Med Phys. 2017;44:902-13.

33. Liu F, Jang $H$, Kijowski $R$, Bradshaw T, McMillan AB. Deep learning MR imaging-based attenuation correction for PET/MR imaging. Radiology. 2017:286:676-84.

34. Fei B, Yang X, Nye JA, Aarsvold JN, Raghunath N, Cervo M, et al. MR/PET quantification tools: registration, segmentation, classification, and MR-based attenuation correction. Med Phys. 2012;39:6443-54.

35. Aitken AP, Giese D, Tsoumpas C, Schleyer P, Kozerke S, Prieto C, et al. Improved UTE-based attenuation correction for cranial PET-MR using dynamic magnetic field monitoring. Med Phys. 2014;41:012302-1-012302-13.

36. Berker Y, Franke J, Salomon A, Palmowski M, Donker HCW, Temur Y, et al. MRI-based attenuation correction for hybrid PET/MRI systems: a 4-class tissue segmentation technique using a combined ultrashort-echo-time/ Dixon MRI sequence. J Nucl Med. 2012;53:796.

37. Wiesinger F, Sacolick LI, Menini A, Kaushik SS, Ahn S, Veit-Haibach P, et al. Zero TE MR bone imaging in the head. Magn Reson Med. 2016;75:107-14. 
38. Khateri P, Rad HS, Jafari AH, Kazerooni AF, Akbarzadeh A, Moghadam MS, et al. Generation of a four-class attenuation map for MRI-based attenuation correction of PET data in the head area using a novel combination of STE/ Dixon-MRI and FCM clustering. Mol Imaging Biol. 2015;17:884-92.

39. Burgos N, Cardoso MJ, Thielemans K, Modat M, Pedemonte S, Dickson J, et al. Attenuation correction synthesis for hybrid PET-MR scanners: application to brain studies. IEEE Trans Med Imaging. 2014;33:2332-41.

40. Izquierdo-Garcia D, Hansen AE, Förster S, Benoit D, Schachoff S, Fürst $S$, et al. An SPM8-based approach for attenuation correction combining segmentation and non-rigid template formation: application to simultaneous PET/MR brain imaging. J Nucl Med Off Publ Soc Nucl Med. 2014;55:1825.

41. Anazodo UC, Thiessen JD, Ssali T, Mandel J, Günther M, Butler J, et al. Feasibility of simultaneous whole-brain imaging on an integrated PET-MRI system using an enhanced 2-point Dixon attenuation correction method. Front Neurosci. 2015:8:434

42. Merida I, Costes N, Heckemann R, Hammers A. Pseudo-CT generation in brain MR-PET attenuation correction: comparison of several multi-atlas methods: EJNMMI physics. Nature Publishing Group; 2015. p. A29. https://ejnmmiphys. springeropen.com/articles/10.1186/2197-7364-2-S1-A29.

43. Sekine T, Buck A, Delso G, Ter Voert EE, Huellner M, Veit-Haibach P, et al. Evaluation of atlas-based attenuation correction for integrated PET/MR in human brain: application of a head atlas and comparison to true CT-based attenuation correction. J Nucl Med. 2016;57:215-20.

44. Torrado-Carvajal A, Herraiz JL, Alcain E, Montemayor AS, Garcia-Cañamaque L, Hernandez-Tamames JA, et al. Fast patch-based pseudo-CT synthesis from T1-weighted MR images for PET/MR attenuation correction in brain studies. J Nucl Med. 2016;57:136-43.

45. Delso G, Carl M, Wiesinger F, Sacolick L, Porto M, Hüllner M, et al. Anatomic evaluation of 3-dimensional ultrashort-echo-time bone maps for PET/MR attenuation correction. J Nucl Med. 2014;55:780-5.

46. Roy S, Wang W-T, Carass A, Prince JL, Butman JA, Pham DL. PET attenuation correction using synthetic $C T$ from ultrashort echo-time MRI. J Nucl Med Off Publ Soc Nucl Med. 2014;55:2071.

47. Poynton CB, Chen KT, Chonde DB, Izquierdo-Garcia D, Gollub RL, Gerstner ER, et al. Probabilistic atlas-based segmentation of combined T1-weighted and DUTE MRI for calculation of head attenuation maps in integrated PET/ MRI scanners. Am J Nucl Med Mol Imaging. 2014:4:160.

48. Navalpakkam BK, Braun H, Kuwert T, Quick HH. Magnetic resonance-based attenuation correction for PET/MR hybrid imaging using continuous valued attenuation maps. Investig Radiol. 2013;48:323-32.

49. Johansson A, Karlsson M, Nyholm T. CT substitute derived from MRI sequences with ultrashort echo time. Med Phys. 2011;38:2708-14.

50. Mehranian A, Arabi H, Zaidi H. Vision 20/20: magnetic resonance imagingguided attenuation correction in PET/MRI: challenges, solutions, and opportunities. Med Phys. 2016;43:1130-55

51. Izquierdo-Garcia D, Catana C. MR imaging-guided attenuation correction of PET data in PET/MR imaging. PET Clin. 2016;11:129-49.

52. Cabello J, Lukas M, Kops ER, Ribeiro A, Shah NJ, Yakushev I, et al. Comparison between MRI-based attenuation correction methods for brain PET in dementia patients. Eur J Nucl Med Mol Imaging. 2016:43:2190-200.

53. Ladefoged CN, Law I, Anazodo U, Lawrence KS, Izquierdo-Garcia D, Catana C, et al. A multi-Centre evaluation of eleven clinically feasible brain PET/MRI attenuation correction techniques using a large cohort of patients. Neurolmage. 2017;147:346-59.

54. Ashburner J, Friston KJ. Unified segmentation. Neurolmage. 2005;26:839-51.

55. Jenkinson M, Beckmann CF, Behrens TEJ, Woolrich MW, Smith SM. Fsl Neuroimage 2012;62:782-790.

56. Prados F, Cardoso MJ, Burgos N, Wheeler-Kingshott CA, Ourselin S. NiftyWeb: web based platform for image processing on the cloud. In: International Society for Magnetic Resonance in Medicine (ISMRM) 24th Scientific Meeting and Exhibition. Singapore; 2016. http://niftyweb.cs.ucl.ac. uk/program.php? $\mathrm{p}=\mathrm{PCT}$ (paragraph publications on $\mathrm{pCT}$ - third point)

57. Nakamoto Y, Osman M, Cohade C, Marshall LT, Links JM, Kohlmyer S, et al. PET/CT: comparison of quantitative tracer uptake between germanium and CT transmission attenuation-corrected images. J Nucl Med. 2002;43:1137-43.

Ready to submit your research? Choose BMC and benefit from:

- fast, convenient online submission

- thorough peer review by experienced researchers in your field

- rapid publication on acceptance

- support for research data, including large and complex data types

- gold Open Access which fosters wider collaboration and increased citations

- maximum visibility for your research: over $100 \mathrm{M}$ website views per year

At BMC, research is always in progress.

Learn more biomedcentral.com/submissions 